\title{
WSI-Mindestlohnbericht 2014 - stagnierende Mindestlöhne
}

Im Jahr 2013 setzt sich in den meisten europäischen Staaten die schwache Mindestlohnentwicklung weiter fort. Lediglich in einigen osteuropäischen Ländern ist eine deutlich stärkere Mindestlohndynamik erkennbar. Angesicht des in vielen europäischen und außereuropäischen Ländern jeweils eher niedrigen Mindestlohnniveaus rückt die Forderung, dieses kräftig zu erhöhen, zunehmend auf die politische Tagesordnung. Eine stärkere Mindestlohndynamik könnte dabei helfen, den akuten Deflationstendenzen in Europa entgegenzuwirken und insgesamt die Einkommenssituation von Niedriglohnempfängern zu verbessern.

THORSTEN SCHULTEN

\section{Einleitung}

Nach dem Koalitionsvertrag der schwarz-roten Bundesregierung soll in Deutschland zum 1. Januar 2015 ein allgemeiner nationaler Mindestlohn von $8,50 €$ pro Stunde eingeführt werden (CDU/CSU/SPD 2013). Damit geht eine mehr als zehn Jahre dauernde Auseinandersetzung zu Ende. Nachdem jetzt die Grundsatzfrage für einen nationalen Mindestlohn in Deutschland entschieden ist, werden sich die hiesigen Kontroversen künftig vermehrt um die konkrete Entwicklung und Gestaltung des Mindestlohns drehen.

Vor diesem Hintergrund ist es sinnvoll, die konkreten Erfahrungen anderer Länder aufzuarbeiten und für die deutsche Debatte nutzbar zu machen. Nationale Mindestlöhne gehören bereits seit Langem in vielen Ländern zu den etablierten Instrumenten bei der Regulierung des Arbeitsmarktes. Nach Angaben der Internationalen Arbeitsorganisation (ILO) verfügen weltweit mehr als 100 Staaten über gesetzliche Mindestlohnregelungen (ILO 2013).

Das Wirtschafts- und Sozialwissenschaftliche Institut (WSI) berichtet seit 2009 im Rahmen seines jährlich erscheinenden WSI-Mindestlohnberichts über die aktuelle Entwicklung von Mindestlöhnen im europäischen und außereuropäischen Ausland. Die Grundlage dieser Berichte bildet die WSI-Mindestlohndatenbank, in der mittlerweile Daten für 30 Länder gespeichert sind. ${ }^{1}$ Hierzu gehö- ren alle 21 von $28 \mathrm{EU}$-Staaten, die über einen nationalen Mindestlohn verfügen. Darüber hinaus enthalten sind auch die Türkei sowie acht weitere außereuropäische Länder (Argentinien, Australien, Brasilien, Japan, Kanada, Korea, Neuseeland und die USA).

\section{Nationale Mindestlöhne zum}

\section{Januar 2014}

Bei der Höhe nationaler Mindestlöhne zeigen sich sowohl innerhalb als auch außerhalb Europas erhebliche Unterschiede. Gemessen in Euro lassen sich im Hinblick auf die Mindestlohnniveaus innerhalb der Europäischen Union (EU) drei Gruppen identifizieren (Abbildung 1): Die erste Gruppe mit relativ hohen Mindestlöhnen umfasst bislang insgesamt sechs Staaten aus Westeuropa. Das höchste Mindestlohnniveau mit einem Wert von $11,10 €$ pro Stunde findet sich in Luxem-

1 Die WSI-Mindestlohndatenbank ist online unter: www.wsi. de/mindestlohndatenbank abrufbar und enthält neben einer interaktiven Karte umfangreicheTabellen und Grafiken. Die Datenbank ist in deutscher und in englischer Sprache verfügbar. 
burg, für dessen Arbeitsmarkt der Mindestlohn angesichts eines extrem hohen Anteils von Berufspendlern aus dem benachbarten Ausland besonders wichtig ist. Der zweithöchste Mindestlohn existiert mit 9,53€ pro Stunde in Frankreich, gefolgt von den Niederlanden mit 9,11€, Belgien mit 9,10€ und Irland mit 8,65€. Mit einem Mindestlohn von 7,43€ pro Stunde bildet Großbritannien das Schlusslicht der europäischen Spitzengruppe. Allerdings wird der in Euro gemessene Betrag des britischen Mindestlohns stark durch den Wechselkurs des britischen Pfunds verzerrt, das seit 2007 gegenüber dem Euro um etwa ein Viertel abgewertet wurde. Ohne diese Abwertung würde der Mindestlohn in Großbritannien heute bei $9,22 €$ pro Stunde liegen und damit einen europäischen Spitzenwert einnehmen. Deutschland würde sich mit dem jetzt anvisierten Mindestlohn von 8,50€ pro Stunde am unteren Rand der westeuropäischen Spitzengruppe bewegen.

In einer zweiten Gruppe mit Mindestlöhnen zwischen $3 €$ und $7 €$ pro Stunde befinden sich insgesamt vier EUStaaten, darunter Slowenien mit 4,56€, Malta mit 4,15€, Spanien mit 3,91 € und Griechenland mit 3,35€. Die dritte Gruppe mit Mindestlöhnen unterhalb von $3 €$ umfasst neben Portugal ausschließlich Länder aus Mittel- und Osteuropa. Das Mindestlohnniveau bewegt sich hier in der Mehrzahl der Länder zwischen 1,50€ und 2,50 € pro Stunde. Lediglich in Bulgarien und Rumänien liegt der Mindestlohn nach wie vor nur knapp über 1,00€ pro Stunde.

Außerhalb der EU hat von den hier berücksichtigten Ländern Australien den höchsten Mindestlohn, der mit $11,88 €$ pro Stunde sogar noch oberhalb des europäischen Spitzenwertes von Luxemburg liegt. Ebenfalls zur obersten Gruppe gehören Neuseeland und Kanada ${ }^{2}$ mit Mindestlöhnen von $8,48 €$ bzw. 7,44 € pro Stunde. Mit Japan, ${ }^{3}$ Korea und den $\mathrm{USA}^{4}$ verfügen drei weitere Länder über ein Mindestlohnniveau, das dem in der europäischen Mittelgruppe entspricht, während Brasilien und Argentinien sich in der unteren europäischen Gruppe wiederfänden. Auch die Türkei verfügt mit 2,17 € über einen Mindestlohn, dessen Niveau mit dem in vielen osteuropäischen Ländern vergleichbar ist.

Die Aussagekraft eines in Euro vorgenommenen Vergleichs absoluter Mindestlohnbeträge ist jedoch begrenzt. Außerhalb der Eurozone wird dieser mitunter stark durch Wechselkursschwankungen verzerrt. Hinzu kommt, dass aufgrund des jeweiligen nationalen Preisniveaus und der damit verbundenen Lebenshaltungskosten den jeweiligen Mindestlohnbeträgen oft eine sehr unterschiedliche Kaufkraft gegenübersteht. Letzterem kann dadurch Rechnung getragen werden, dass der internationale Vergleich gesetzlicher Mindestlöhne zusätzlich in Kaufkraftstandards (KKS) durchgeführt wird (Abbildung 2). Gegenüber dem nominellen Mindestlohnvergleich in Euro werden die Niveauunterschiede zwischen den nationalen Mindestlöhnen bei einem Vergleich in KKS deutlich kleiner: Während die Differenz zwischen dem höchsten und dem niedrigsten Min-
ABB. 1

\section{Nationale Mindestlöhne pro Stunde 2014*}
Angaben in Euro
unter $3 €$
3 bis $7 €$
mehr als $7 €$

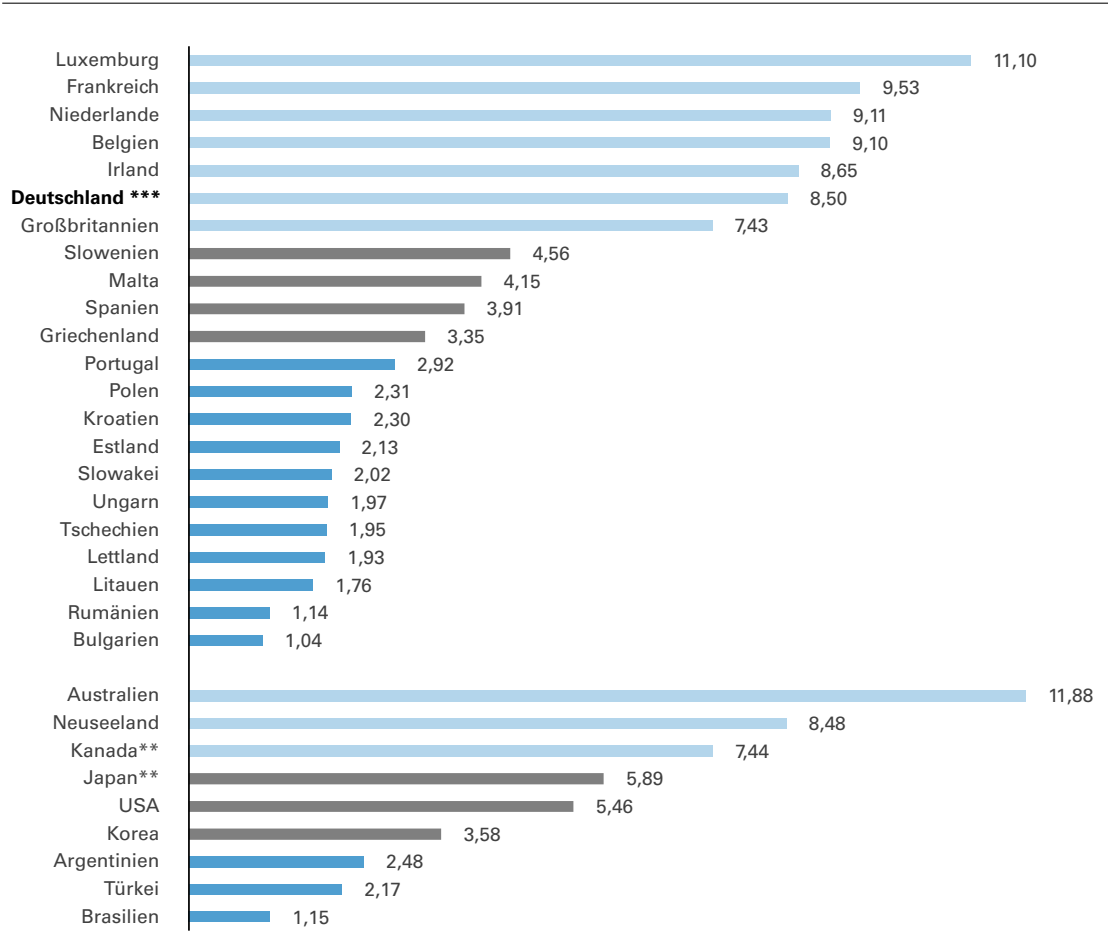

* Stand Januar 2014; Umrechnung in Euro zum Jahresdurchschnittskurs 2013.

** gewichteter Durchschnitt regionaler Mindestlöhne.

***ab dem 01.01.2015.

Quelle:WSI-Mindestlohndatenbank 2014

destlohn gemessen in Euro bei etwa 1:11 liegt, ist sie gemessen in KKS mit einem Verhältnis von 1:6 deutlich geringer.

Bei der Berechnung in KKS kommt es zum Teil auch zu Veränderungen in der Rangfolge der Staaten. So

2 In Kanada werden die Mindestlöhne auf der Ebene der Provinzen festgelegt. Da kein nationaler Mindestlohn existiert, wird hier ein nach Beschäftigtenzahl gewichteter nationaler Durchschnittswert verwendet, der Anfang 2014 bei umgerechnet 7,44 € lag. Der höchste Mindestlohn existierte in der Provinz Nunavut mit 8,04€, während die Provinz Alberta mit 7,27 € den niedrigsten Mindestlohnwert aufwies.

3 Ähnlich wie in Kanada existiert auch in Japan kein einheitlicher nationaler Mindestlohn. Mindestlöhne werden vielmehr auf regionaler Ebene festgelegt. Gegenüber dem gewichteten nationalen Durchschnittswert liegen die regionalen Mindestlöhne jeweils bis zu $14 \%$ darüber oder darunter. Der höchste japanische Mindestlohn gilt in Tokyo mit umgerechnet $6,70 €$.

4 Zusätzlich zum nationalen Mindestlohn existieren in den USA auch regionale Mindestlöhne auf der Ebene der USBundesstaaten. In 20 von 50 Bundesstaaten liegt der Mindestlohn dabei oberhalb der nationalen Mindestlohngrenze. Den höchsten Wert weist der Bundesstaat Washington mit umgerechnet 7,02 € pro Stunde auf. 
ABB. 2

\section{Nationale Mindestlöhne pro Stunde 2014*}

Angaben in Kaufkraftstandards (KKS)* $\quad$ unter 3 KKS $\quad 3$ bis 7 KKS mehr als 7 KKS

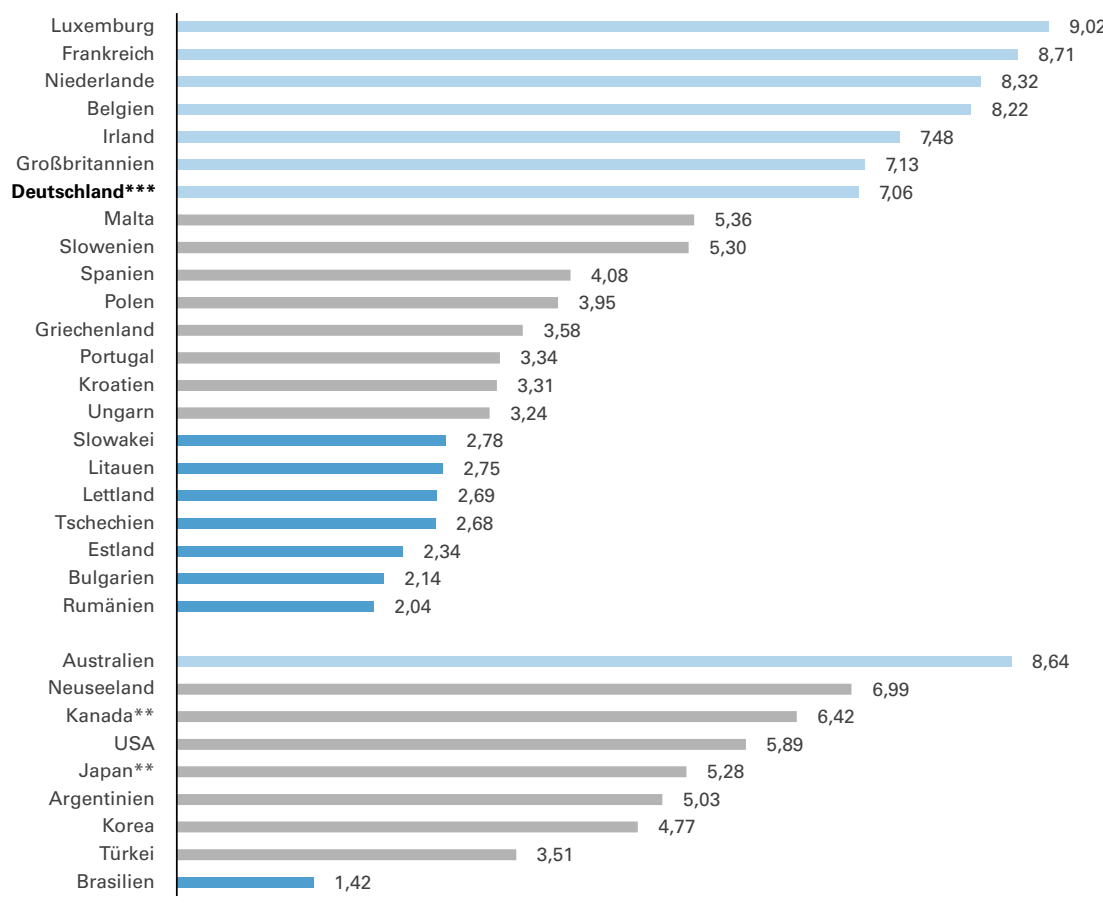

Stand Januar 2014; Umrechnung in KKS aufgrund der von der Weltbank für 2012

ausgewiesenen Kaufkraftparitäten für den privaten Konsum.

** Gewichteter Durchschnitt regionaler Mindestlöhne.

*** Ausgehend von einem Mindestlohn von 8,50 € (ab 01.01.2015).

Quelle:WSI-Mindestlohndatenbank 2014.

fällt z. B. Australien mit dem auf Euro-Basis höchsten Mindestlohn gemessen in KKS noch hinter die Werte von Luxemburg und Frankreich zurück. Deutschland würde hingegen mit einem Mindestlohn von 8,50€ kaufkraftbereinigt noch unterhalb des Mindestlohnniveaus von Großbritannien liegen. Insgesamt weisen die Mindestlöhne jedoch auch nach einer Umrechnung in KKS erhebliche nationale Niveauunterschiede auf und reflektieren damit das sowohl in Europa als auch im außereuropäischen Raum bestehende Lohngefälle.

\section{Der relative Wert gesetzlicher Mindestlöhne (Kaitz-Index)}

Die Bedeutung des Mindestlohns wird nicht allein durch seinen absoluten Wert bestimmt, sondern auch durch seine Stellung im jeweiligen nationalen Lohngefüge. Letztere kann durch den sogenannten Kaitz-Index (benannt nach dem amerikanischen Ökonomen Hyman Kaitz) bestimmt werden, der den relativen Wert des gesetzlichen Mindestlohns zum Ausdruck bringt und statistisch als Prozentsatz des Mindestlohns vom jeweiligen nationalen Durchschnitts- oder Medianlohn gemessen wird. Während der Durchschnittslohn durch das arithmetische Mittel aller Löhne bestimmt wird, ist der Medianlohn der mittlere Lohn, bei dem die Hälfte aller Beschäftigten mehr und die andere Hälfte weniger verdient.

Im Folgenden wird bei der Analyse des Kaitz-Index auf die OECD-Einkommensdatenbank zurückgegriffen, die Berechnungen zum Anteil des nationalen Mindestlohns am jeweiligen nationalen Medianlohn für Vollzeitbeschäftigte enthält (Abbildung 3). ${ }^{5}$ Für das Jahr 2012 (die Daten für 2013 liegen nocht nicht vor) zeigt der Kaitz-Index eine große Schwankungsbreite. Von den insgesamt 25 Ländern, für die entsprechende Daten vorliegen, bewegte sich der Mindestlohn in der größten Gruppe von 12 Staaten zwischen 40 und $49 \%$ des Medianlohns. Unterhalb dieses Niveaus befanden sich mit Japan, den USA, Estland und der Tschechischen Republik vier Staaten, deren relativer Mindestlohnwert 36 bzw. $38 \%$ des jeweiligen Medianlohns betrug.

Fünf weitere Staaten (Portugal, Ungarn, Australien, Belgien und Lettland) verfügten hingegen über ein deutlich höheres Mindestlohnniveau zwischen 50 und $59 \%$ des nationalen Medianlohns. Die gemessen am Medianlohn höchsten Mindestlöhne existierten mit Werten von $60 \%$ und mehr in Frankreich, Neuseeland und Slowenien sowie als Ausreißer nach oben der Türkei mit $73 \%$. Hätte Deutschland bereits im Jahr 2012 einen Mindestlohn von 8,50€ pro Stunde eingeführt, so würde dieser bei $51 \%$ des Medianlohns für Vollzeitbeschäftigte liegen. ${ }^{6}$

Die Analyse des Kaitz-Index macht insgesamt deutlich, dass das relative Niveau des Mindestlohns in den meisten Fällen nicht besonders hoch ist. Mit Ausnahme der Türkei liegt der Mindestlohn überall unterhalb der offiziellen Niedriglohnschwelle, die nach internationalen Konventionen bei zwei Dritteln des Medianlohns angesetzt wird. In der Mehrzahl der hier erfassten Länder liegt der Mindestlohn sogar unterhalb der 50 \%-Schwelle des nationalen Medianlohns und muss somit als Armutslohn klassifiziert werden (Marx et al. 2012). Vor diesem Hintergrund wird in vielen europä-

5 Die entsprechenden Daten finden sich unter: https://stats. oecd.org/Index.aspx?DataSetCode=MIN2AVE. Die Betrachtung des Medianlohns hat gegenüber dem Durchschnittslohn den Vorteil, dass er statistisch besser zu erfassen ist und zugleich das mittlere Lohngefüge einer Gesellschaft besser beschreibt. Die in der Regel jeweils deutlich oberhalb des Medianlohns liegenden Durchschnittslöhne werden hingegen vor allem durch extrem hohe Löhne im oberen Lohnsegment nach oben gedrückt. Die OECD-Datenbank basiert auf nicht-harmonisierten nationalen Datenquellen, sodass der hier ermittelte Kaitz-Index eher als Näherungswert gelesen werden muss.

6 Die Beschäftigungsstatistik der Bundesagentur für Arbeit weist für das Jahr 2012 für Vollzeitbeschäftigte einen Medianlohn von $2.889 €$ pro Monat aus. Bei einer 40-Stunden-Woche entspricht dies einem Median-Stundenlohn von 16,70 €. Ein Mindestlohn von 8,50€ pro Stunde läge demnach bei $50,9 \%$ des Medianlohns. 
ischen und außereuropäischen Ländern derzeit vonseiten der Gewerkschaften und anderer sozialer Bewegungen eine deutliche Erhöhung des Mindestlohns gefordert. In eine ähnliche Richtung zielen auch Debatten über eine europäische Mindestlohnpolitik, in denen vorgeschlagen wird, den Mindestlohn z. B. auf $60 \%$ des jeweiligen nationalen Medianlohns anzuheben (Schulten 2012; Fernández-Macías/Vacas-Soriano 2013)

\section{Die aktuelle Entwicklung der Mindestlöhne im Jahr 2013}

Seit Beginn des Jahres 2013 wurden in insgesamt 23 der hier untersuchten 30 Staaten nominale Erhöhungen der Mindestlohnsätze vorgenommen (Abbildung 4). Hiervon haben allein 18 Staaten ihre Mindestlöhne zuletzt zum 1. Januar 2014 erhöht. In sieben Ländern gab es dagegen keine Mindestlohnzuwächse, sodass die bestehenden nominalen Mindestlohnbeträge eingefroren wurden.

Im Hinblick auf die Entwicklungsdynamik der Mindestlöhne lassen sich für 2013 insgesamt vier Gruppen unterscheiden. Die größte Gruppe umfasst zehn Staaten, in denen die Erhöhung relativ moderat ausfiel und sich zwischen 0,7 und 2,6 \% bewegte. $\mathrm{Zu}$ dieser Gruppe gehören vor allem westeuropäische Länder wie Großbritannien, Frankreich, die Niederlande und Luxemburg. Hinzu kommen Malta und Slowenien sowie außerhalb Europas Australien, Neuseeland und Kanada.

Eine zweite, mit neun Staaten ebenfalls recht große Gruppe zeichnet sich mit Werten zwischen 3,5 und 9,7\% durch deutlich kräftigere Mindestlohnerhöhungen aus. Zu dieser Gruppe gehören ausschließlich osteuropäische Länder sowie außerhalb Europas Brasilien und Korea. Gemeinsam ist dieser Gruppe, dass sie allesamt über ein relativ geringes Mindestlohnniveau verfügen, sodass die hohen Steigerungsraten nach wie vor durch starke Basiseffekte beeinflusst werden.

Eine dritte Gruppe umfasst vier weitere Staaten, in denen die nominalen Mindestlohnsätze im Laufe des Jahres 2013 sogar um zweistellige Werte erhöht worden sind. Einsamer Spitzenreiter war hierbei Argentinien mit einem Mindestlohnzuwachs von $34,8 \%$, gefolgt von Rumänien mit $21,4 \%$, Lettland mit 12,9\% und Estland mit 12,1 \%. Schließlich gibt es eine vierte Gruppe, in der es 2013 keine Mindestlohnerhöhungen gegeben hat. Zu dieser Gruppe gehören neben den USA sechs Länder aus der EU, darunter Belgien und Litauen sowie die europäischen Krisenstaaten Griechenland, Portugal, Spanien und Irland.

Die unterschiedliche Entwicklung der nominalen Mindestlohnsätze reflektiert zum Teil die unterschiedlichen Preisdynamiken in den jeweiligen Ländern. Vor diesem Hintergrund ist es wichtig, die reale, also um den Zuwachs um der Verbraucherpreise bereinigte Entwicklung der Mindestlöhne zu analysieren (Abbildung 5). Offensichtlich ist der
ABB. 3

\section{Der relative Wert des Mindestlohns 2012}

Nationaler Mindestlohn in \% des nationalen Medianlohns für Vollzeitbeschäftigte Angaben in Prozent

- $60 \%$ und mehr 50 bis $59 \%$
40 bis $49 \%$

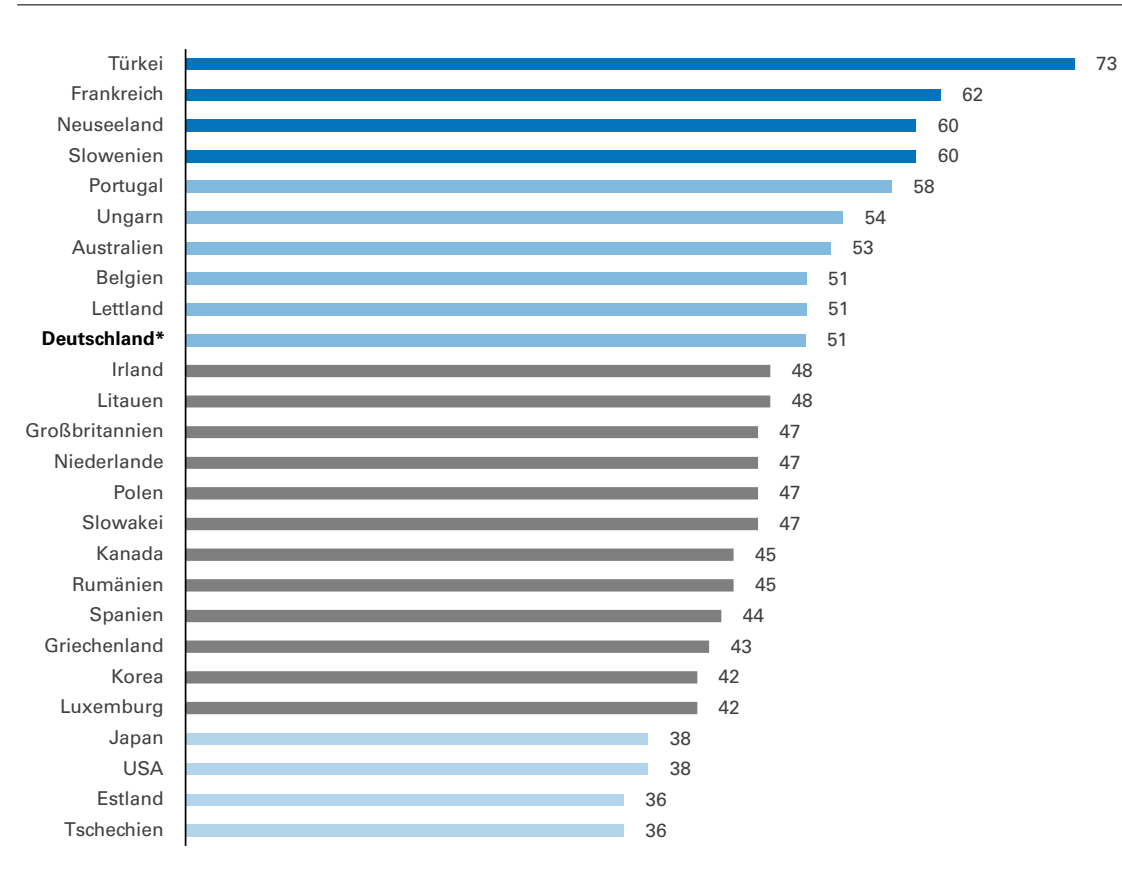

*Ausgehend von einem fiktiven Mindestlohn von 8,50€ pro Stunde.

Quelle: OECD; für Deutschland: Berechnungen des WSI auf der Grundlage von Daten der Beschäftigungsstatistik der Bundesagentur für Arbeit.

außergewöhnlich hohe Anstieg des Mindestlohns in Argentinien Ausdruck eines extrem inflationären Umfeldes mit offiziellen Preissteigerungsraten von mehr als $10 \%$. Die immer noch hohen offiziellen Reallohnzuwächse beim Mindestlohn von fast $24 \%$ stehen hingegen zum einen für die Erwartung weiterer Preissteigerungen. Zum anderen tragen sie der Tatsache Rechnung, dass die offiziellen Daten die tatsächliche Inflationsrate deutlich unterschätzen und damit auch die tatsächlichen Reallohnzuwächse deutlich geringer ausfallen.

Anders sieht es bei den osteuropäischen Staaten Rumänien, Lettland und Estland aus, wo der Preisanstieg deutlich geringer ausfiel und die hohen Reallohnzuwächse zwischen 9,3 und 17,4 \% beim Mindestlohn eher für einen lohnpolitischen Nachholprozess nach einer schwachen Mindestlohnentwicklung in den Vorjahren stehen. In Lettland ist angesichts eines eher deflationären Umfeldes mit einer stagnierenden Preisentwicklung der Nominalzuwachs von $12,9 \%$ sogar vollständig als Reallohnzuwachs realisiert worden.

Auch in anderen osteuropäischen Ländern konnten, gestützt auf eher rückläufige Preissteigerungsraten, bei den Mindestlöhnen relativ hohe Reallohnzuwächse zwischen knapp 3 und $9 \%$ realisiert werden. Hierzu gehören Län- 
ABB.

\section{Nominale Entwicklung gesetzlicher Mindestlöhne 2013*}

Angaben in Prozent

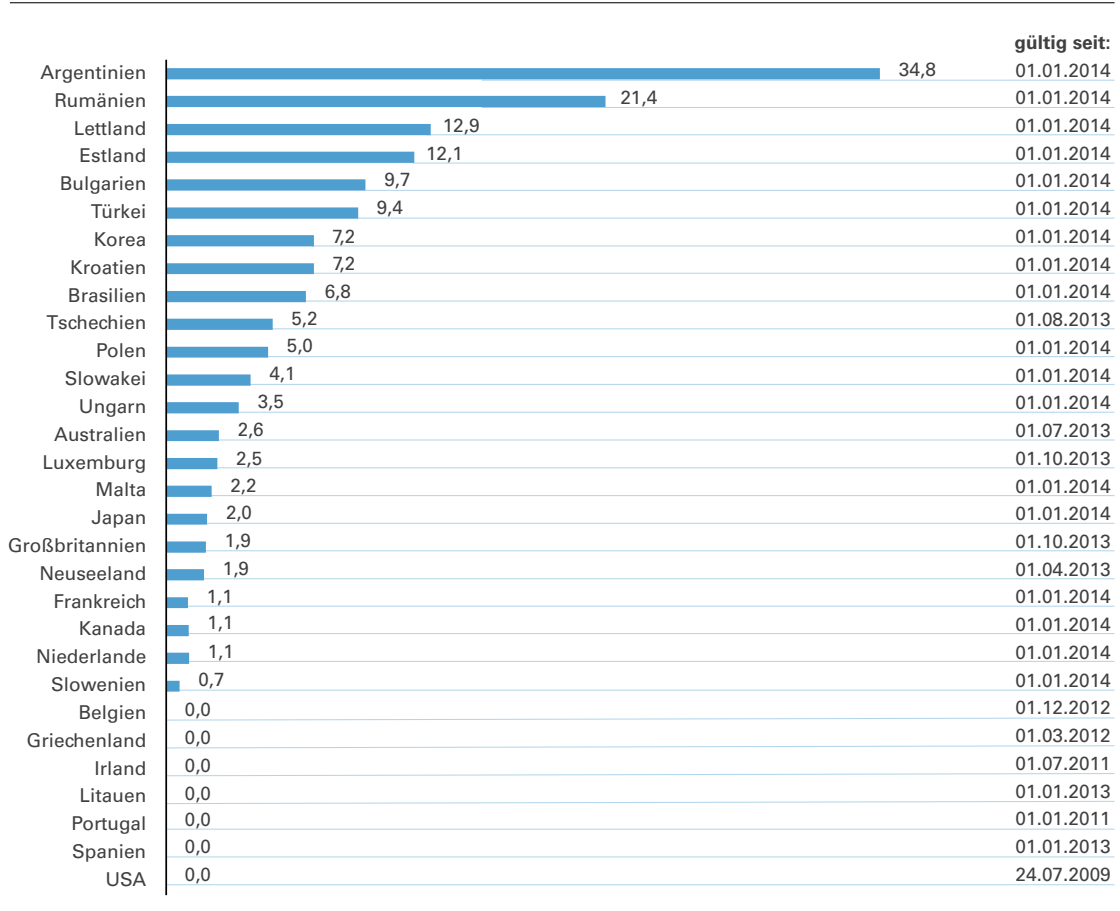

*Entwicklung vom 01.01.2013 zum 01.01.2014

Quelle: WSI-Mindestlohndatenbank 2014.

\section{Langfristige Entwicklungstrends bei den Mindestlöhnen}

Seit Beginn der 2000er Jahre zeigt die Entwicklung der Mindestlöhne in den meisten der analysierten Länder einen äußerst moderaten Verlauf (Tabelle 1). Dies gilt insbesondere für die Länder West- und Südeuropas. Der Mindestlohn stieg hier preisbereinigt im Jahresdurchschnitt zwischen 0,1 \% in Belgien und 1,9\% in Großbritannien. In Griechenland und den Niederlanden lässt sich über die letzten 13 Jahre hinweg sogar ein leichter Rückgang des realen Mindestlohnwertes feststellen, wobei dies in Griechenland vor allem auf die durch die Troika aus Europäischer Kommission, Europäischer Zentralbank und Internationalem Währungsfonds erzwungene starke Kürzung des Mindestlohns im Jahr 2012 zurückzuführen ist (Schulten 2013).

Auch in vielen außereuropäischen Staaten wie Australien, Japan, Kanada oder den USA kam es beim Mindestlohn lediglich zu moderaten Reallohnzuwächsen von jahresdurchschnittlich weniger als $2 \%$ (Neuseeland liegt knapp oberhalb dieses Wertes). Das Schlusslicht bildet die USA, deren realer Mindestlohnbetrag seit $2001 \mathrm{im}$ Durchschnitt pro Jahr nur um $0,4 \%$ zunahm.

Eine deutlich stärkere Mindestlohndynamik lässt sich dagegen in einigen osteuropäischen Ländern sowie in Argentinien, Brasilien und Korea beobachten. In diesen Ländern nahm der preisbereinigte Mindestlohn jahresdurchschnittlich zwischen 3 und $9 \%$ - in Argentinien sogar um 16,2 \% - zu. Diese deutlich höheren Mindestlohnzuwächse sind zum wesentlichen Teil Ausdruck von Basiseffekten, die auf einem in absoluten Zahlen immer noch sehr niedrigem Mindestlohnniveau beruhen. Sie stehen zudem für Auf- und Nachholprozesse bei der allgemeinen Lohnentwicklung in diesen Ländern. Insbesondere im Fall von Argentinien werden sie zudem durch eine rasante Preisentwicklung geprägt, die durch die offiziellen Inflationsraten noch unterschätzt wird.

Bei der langfristigen Betrachtung wird insgesamt deutlich, dass die Entwicklung der Mindestlöhne in der Regel eng an den Konjunkturverlauf gebunden ist. Während in den Aufschwungjahren bis Mitte der 2000er Jahre eher stärkere Mindestlohnsteigerungen erfolgten, sind die Mindestlohnanpassungen seit der Krise 2008ff. in vielen Ländern hinter der Inflationsentwicklung zurückgeblieben und haben damit zu einem erheblichen Reallohnverlust bei den Mindestlohnempfängern geführt. In vielen Ländern, wie z. B. Irland, Portugal, Spanien und Griechenland, wurde diese Entwicklung zudem durch politische Interventionen der EU verstärkt, die darauf drängte, die Mindestlöhne einzufrieren oder sogar zu kürzen (Schulten/Müller 2013).

Schließlich wird die Entwicklung der Mindestlöhne auch durch die unterschiedlichen Regeln und Verfahren der Mindestlohnanpassung beeinflusst (Schulten 2014). Idealtypisch kann dies am Beispiel Frankreichs, Großbritanniens und den USA aufgezeigt werden (Abbildung 6). In Frankreich hat die Krise 2008ff. zwar zu einer deutlichen Abflachung der Min- 
destlohndynamik geführt, durch die gesetzlich vorgeschriebene Mindestanpassung des Mindestlohns an die Preisentwicklung konnte jedoch sichergestellt werden, dass der reale Wert des Mindestlohns erhalten bleibt.

Einen völlig anderen Verlauf nahm dagegen die Entwicklung in Großbritannien, wo der Mindestlohn jährlich auf Empfehlung der sogenannten Low Pay Commission (aus Vertretern der Arbeitgeber, Gewerkschaften und Wissenschaft) von der Regierung angepasst wird. Nachdem die Entwicklung des britischen Mindestlohns auf der Grundlage eines sehr niedrigen Ausgangsniveaus in der ersten Hälfte der 2000er Jahre zunächst sehr hohe Zuwachsraten aufwies, ist sie mittlerweile seit nunmehr fünf Jahren in Folge hinter der Inflationsrate zurückgeblieben. Der reale Wert des britischen Mindestlohns liegt demnach heute noch unterhalb des Wertes des Jahres 2005.

Eine wiederum andere Verlaufsform hat die Entwicklung des Mindestlohns in den USA, wo keine vorgegebenen Regeln für die Anpassung des Mindestlohns bestehen. Zwischen 2000 und 2006 wurde hier der nationale Mindestlohn eingefroren, sodass er real kontinuierlich an Wert verlor. Zwischen 2007 und 2009 kam es dann kurzfristig zu einer relativ starken Anhebung, während seit 2009 das Mindestlohnniveau erneut stagniert.

\section{Ausblick: Höhere Mindestlöhne auf der Tagesordnung}

Die Entwicklung des Mindestlohns wird nach wie vor von den Folgewirkungen der in den meisten Ländern noch nicht überwundenen Krise 2008ff. geprägt. In vielen Ländern wurden die Mindestlöhne eingefroren oder blieben deutlich hinter der Inflationsentwicklung zurück. Damit sank der reale Wert des Mindestlohns immer weiter nach unten. Hinzu kommt, dass das Niveau des Mindestlohns auch vor der Krise mitunter nicht besonders hoch war und in vielen Fällen die Armutsschwelle nicht überschritten hat.

Deswegen mehren sich vielerorts die politischen Stimmen, die für eine deutlich höhere Anhebung des Mindestlohns plädieren. In einigen osteuropäischen Staaten, wie z. B. den baltischen Ländern oder Rumänien, ist es bereits in den vergangen beiden Jahren wieder zu einer deutlich stärkeren Anhebung der Mindestlöhne gekommen. Besonders intensive Debatten über eine kräftige Anhebung des Mindestlohns werden derzeit vor allem in Großbritannien und den USA geführt (Financial Times Editorial 2014).

In Großbritannien haben mittlerweile mehrere Studien auf den anhaltenden Wertverlust des britischen Mindestlohns und die damit einhergehenden sozialen Probleme aufmerksam gemacht (z. B. Plunkett/Hurrell 2013). Vor dem Hintergrund des sehr niedrigen britischen Mindestlohnniveaus
ABB. 5

Reale Entwicklung gesetzlicher Mindestlöne 2013*

Angaben in Prozent

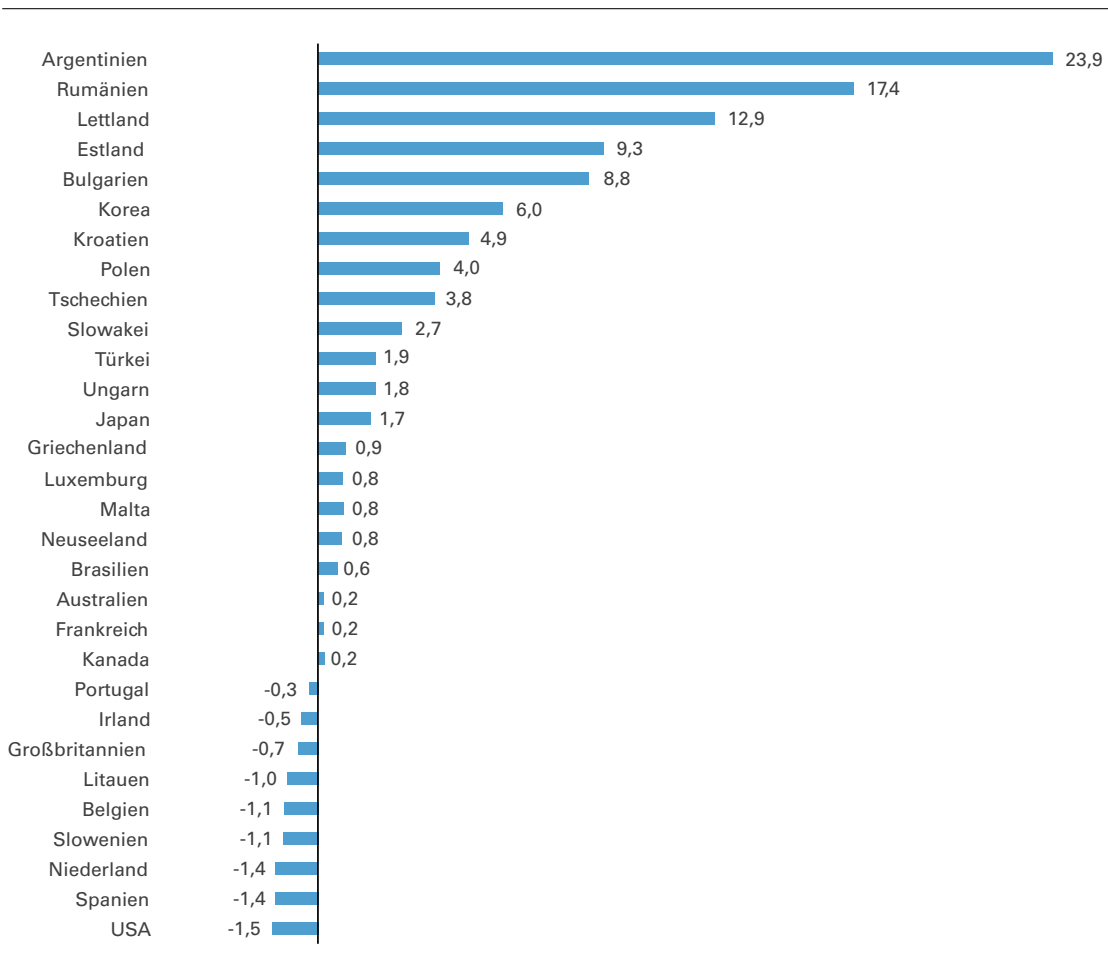

*Entwicklung vom 01.01.2013 zum 01.01.2014; nominale Erhöhung deflationiert um den nationalen Anstieg der Verbraucherpreise 2013.

Quelle:WSI-Mindestlohndatenbank 2014

\section{ABB. 6}

Entwicklung des realen Mindestlohns in Frankreich, Großbritannien und den USA 2000 - 2013*

$2000=100$ - Großbritannien - Frankreich - USA

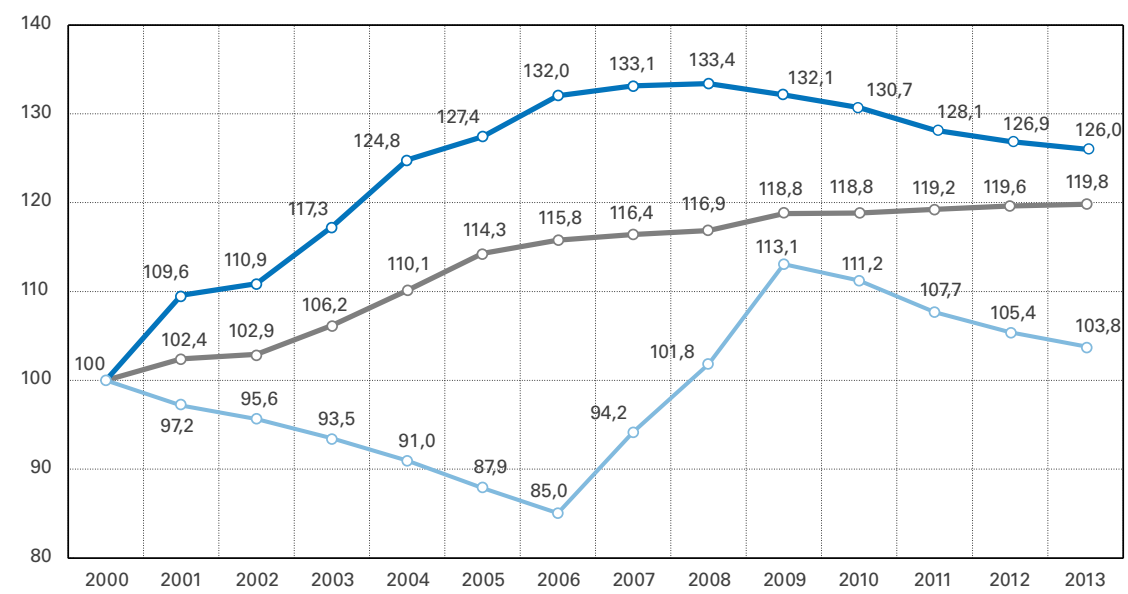

*Entwicklung des nominalen Mindestlohns deflationiert um die Entwicklung de Verbraucherpreise.

Quelle:WSI-Mindestlohndatenbank 2014 
TABELLE 1

Entwicklung der realen Mindestlöhne 2001-2013*

Angaben in Prozent zum Vorjahr

\begin{tabular}{|c|c|c|c|c|c|c|c|c|c|c|c|c|c|c|}
\hline & 2001 & 2002 & 2003 & 2004 & 2005 & 2006 & 2007 & 2008 & 2009 & 2010 & 2011 & 2012 & 2013 & $\begin{array}{c}2001- \\
2013^{* *}\end{array}$ \\
\hline \multicolumn{15}{|c|}{ West- und Südeuropa } \\
\hline Belgien & 1,5 & 0,4 & $-1,6$ & $-0,1$ & $-0,8$ & 0,2 & 2,2 & 1,5 & 0,1 & $-0,2$ & $-1,6$ & 1,2 & $-1,1$ & 0,1 \\
\hline Frankreich & 2,4 & 0,5 & 3,2 & 3,7 & 3,8 & 1,3 & 0,6 & 0,4 & 1,6 & 0,1 & 0,3 & 0,3 & 0,2 & 1,4 \\
\hline Griechenland & 1,9 & 2,5 & 0,3 & 3,0 & 2,7 & $-0,2$ & 5,9 & $-1,2$ & 4,4 & $-4,7$ & $-1,9$ & $-24,3$ & 0,9 & $-0,8$ \\
\hline Großbritannien & 9,6 & 1,2 & 5,8 & 6,4 & 2,1 & 3,6 & 0,8 & 0,2 & $-1,0$ & $-1,1$ & $-2,0$ & $-1,0$ & $-0,7$ & 1,9 \\
\hline Irland & 1,9 & 1,7 & $-3,5$ & 8,0 & 6,9 & 4,6 & $-0,7$ & $-4,1$ & 4,5 & 0,9 & $-2,6$ & $-1,7$ & $-0,5$ & 1,2 \\
\hline Luxemburg & $-0,2$ & 4,0 & 0,5 & 2,3 & 0,0 & 1,8 & $-2,3$ & 1,1 & 2,2 & 2,1 & $-0,9$ & 1,4 & 0,8 & 1,0 \\
\hline Malta & 0,1 & 1,2 & 0,1 & 0,5 & 1,0 & 0,2 & 1,3 & $-1,4$ & 1,9 & $-0,9$ & 0,4 & 0,1 & 0,8 & 0,4 \\
\hline Niederlande & 0,4 & 0,2 & $-0,9$ & $-1,2$ & $-1,1$ & 1,0 & 1,0 & 1,0 & 0,8 & $-0,1$ & $-0,7$ & $-1,0$ & $-1,4$ & $-0,2$ \\
\hline Portugal & $-0,1$ & $-1,1$ & $-0,7$ & 0,1 & 0,7 & 1,3 & 3,3 & 3,1 & 6,4 & 0,7 & $-3,7$ & $-2,8$ & $-0,3$ & 0,5 \\
\hline Spanien & $-1,7$ & $-1,0$ & $-1,0$ & 8,4 & 2,1 & 2,0 & 2,4 & $-0,1$ & 1,8 & $-0,5$ & $-3,2$ & $-1,9$ & $-1,4$ & 0,4 \\
\hline \multicolumn{15}{|c|}{ Osteuropa } \\
\hline Bulgarien & 18,1 & 4,4 & 7,0 & 19,1 & 1,7 & 5,3 & 13,1 & $-3,1$ & $-2,8$ & $-2,4$ & 9,2 & 11,9 & 8,8 & 7,0 \\
\hline Estland & 9,0 & 13,6 & 13,3 & 5,5 & 7,7 & 15,7 & 19,7 & $-10,4$ & 0,1 & $-3,0$ & $-0,9$ & 1,6 & 9,3 & 6,2 \\
\hline Kroatien & 2,0 & 1,7 & 3,1 & 4,6 & 0,9 & 2,7 & 3,4 & 6,4 & 0,1 & $-1,0$ & $-2,3$ & $-3,4$ & 4,9 & 1,8 \\
\hline Lettland & 18,1 & 15,5 & 10,1 & $-6,2$ & 6,2 & 26,8 & 24,8 & $-2,8$ & $-3,5$ & 10,9 & $-4,4$ & $-1,1$ & 12,9 & 8,2 \\
\hline Litauen & $-1,4$ & $-0,3$ & 6,6 & 9,3 & 10,9 & 5,2 & 27,2 & $-10,9$ & $-4,4$ & $-1,3$ & $-4,1$ & 21,8 & $-1,0$ & 4,4 \\
\hline Polen & $-5,3$ & 3,4 & 2,3 & $-0,3$ & 3,7 & 2,8 & 17,9 & 9,2 & $-0,6$ & 2,6 & 4,0 & 3,1 & 4,0 & 3,6 \\
\hline Rumänien & 5,5 & 56,1 & $-3,3$ & $-1,2$ & $-2,5$ & 11,6 & 23,4 & 12,2 & $-5,6$ & 5,6 & $-1,3$ & $-3,3$ & 17,4 & 8,8 \\
\hline Slowakei & 4,4 & 17,2 & 0,8 & $-0,7$ & 3,4 & 5,6 & 3,9 & 5,2 & 2,6 & 1,9 & $-0,6$ & $-0,4$ & 2,7 & 3,5 \\
\hline Slowenien & 8,7 & 2,0 & 2,0 & 1,8 & 1,9 & $-0,5$ & $-0,4$ & 3,8 & 0,4 & 23,4 & 0,3 & 0,1 & $-1,1$ & 3,3 \\
\hline Tschechien & 8,3 & 7,0 & 7,2 & 4,5 & 3,3 & 5,1 & $-3,0$ & $-6,3$ & $-1,0$ & $-1,5$ & $-1,9$ & $-3,3$ & 3,8 & 1,7 \\
\hline Türkei & 4,2 & $-7,1$ & 16,6 & 6,9 & 0,5 & $-3,7$ & $-0,6$ & $-1,0$ & 3,2 & 0,6 & 5,0 & 1,4 & 1,9 & 2,2 \\
\hline Ungarn & 16,1 & $-5,3$ & 1,2 & 0,8 & 6,2 & 0,8 & $-2,7$ & $-2,5$ & $-1,3$ & 1,3 & 15,3 & $-0,3$ & 1,8 & 2,4 \\
\hline \multicolumn{15}{|c|}{ Außerhalb Europas } \\
\hline Argentinien & 1,1 & $-25,9$ & 61,6 & 24,2 & 30,4 & 16,1 & 13,7 & 17,9 & 14,7 & 11,9 & 15,5 & 5,3 & 23,9 & 16,2 \\
\hline Australien & 4,4 & 4,7 & 1,2 & 1,9 & 1,0 & 2,1 & $-0,3$ & $-0,2$ & $-1,8$ & 1,9 & 0,1 & 1,1 & 0,2 & 1,3 \\
\hline Brasilien & 12,0 & 2,5 & 5,1 & 1,7 & 8,4 & 12,7 & 5,2 & 3,5 & 17,9 & 0,6 & 8,9 & 3,4 & 0,6 & 6,3 \\
\hline Japan & 1,6 & 0,9 & 0,2 & 0,2 & 0,7 & 0,5 & 2,0 & 1,0 & 2,8 & 3,1 & 1,3 & 1,6 & 1,7 & 1,4 \\
\hline Kanada & $-1,2$ & $-0,9$ & $-2,1$ & 0,6 & 2,3 & 0,8 & 2,1 & 4,4 & 5,9 & 3,6 & 0,8 & 0,7 & 0,2 & 1,3 \\
\hline Korea & 8,5 & 5,6 & 6,8 & 9,6 & 6,4 & 10,0 & 5,8 & 1,4 & 0,0 & 2,2 & 2,0 & 3,9 & 6,0 & 5,2 \\
\hline Neuseeland & $-0,6$ & 1,2 & 4,5 & 3,6 & 2,5 & 4,5 & 7,4 & 2,7 & 2,1 & $-0,3$ & $-2,0$ & 2,7 & 0,8 & 2,2 \\
\hline USA & $-2,8$ & $-1,6$ & $-2,3$ & $-2,7$ & $-3,4$ & $-3,2$ & 10,7 & 8,1 & 11.0 & $-1,6$ & $-3,2$ & $-2,1$ & $-1,5$ & 0,4 \\
\hline
\end{tabular}

* nominale Entwicklung der Mindestlöhne vom 1. Januar eines Jahres zum 1. Januar des Folgejahres deflationiert um die Entwicklung der Verbraucherpreise.

** durchschnittliche Entwicklung pro Jahr.

Quelle: WSI Mindestlohndatenbank 2014. 
sind, ausgehend von London, überall im Land sogenannte „Living-Wage Initiativen" entstanden, die auf lokaler Ebene für einen höheren existenzsichernden Mindestlohn eintreten, der über den öffentlichen Dienst, die öffentliche Auftragsvergabe und freiwillige Selbstverpflichtungen von Unternehmen durchgesetzt werden soll. ${ }^{7}$ In London hat die Stadtregierung im November 2013 beschlossen, den „London Living Wage“ auf $£ 8,80(10,36 €)$ anzuheben (Greater London Authority 2013). Damit liegt er fast $40 \%$ oberhalb des gesetzlichen Mindestlohns in Großbritannien. Außerhalb Londons treten die lokalen Living-Wage-Initiativen für einen existenzsichernden Mindestlohn von $£ 7,65$ (9,01 $€$ ) ein, der damit immer noch $21 \%$ über dem gesetzlichen Mindestlohn liegt. Vor diesem Hintergrund haben mittlerweile sogar Vertreter der konservativen britischen Regierung dafür plädiert, 2014 den Mindestlohn deutlich stärker zu erhöhen.

In den USA hat der anhaltende Wertverlust des nationalen Mindestlohns dazu geführt, dass immer mehr Bundesstaaten ihre regionalen Mindestlöhne über das nationale Niveau angehoben haben. Bereits seit Längerem wird von Ökonomen auf die unzureichende Anpassung des nationalen Mindestlohns hingewiesen. In einem offenen Appell haben fast 600 US-Ökonomen (darunter zahlreiche Nobelpreisträger) die amerikanische Regierung aufgefordert, den bisherigen Wertverlust des Mindestlohns wieder auszugleichen und den nationalen Mindestlohn auf \$ 10,10 (7,60 €) zu erhöhen, was einer Anhebung um fast $40 \%$ entsprechen würde (Aaron et al. 2013). Bereits im März 2013 hatten Abgeordnete der Demokratischen Partei einen Gesetzentwurf vorgelegt, der in drei Schritten eine Erhöhung des Mindestlohns auf $\$ 10,10$ vorsieht und danach eine regelmäßige Anpassung des Mindestlohns an die Inflationsrate einführen will. ${ }^{8}$ Während US-Präsident Obama diesen Vorschlag unterstützte, scheiterte seine Umsetzung bislang an der republikanischen Mehrheit im Repräsentantenhaus.

In Europa drängt dagegen vor allem die EU immer noch auf eine eher restriktive Mindestlohnpolitik, die in verschiedenen Ländern das Vorgehen einschließt, die Mindestlöhne einzufrieren oder gar zu kürzen (Schulten/ Müller 2013). Angesicht der immer deutlicher zutage tretenden Deflationsgefahren in ganz Europa wäre hingegen genau die entgegengesetzte Politik notwendig. Nur durch deutlich höhere Lohnsteigerungen ließe sich der anhaltenden Nachfrageschwäche in Europa entgegentreten, sodass der Mindestlohn seine Funktion als „Deflationsbremse“ (Herr et al. 2009) wahrnehmen kann. Vor diesem Hintergrund ist die Frage, welche Entwicklungsdynamik der neue Mindestlohn in Deutschland nehmen wird, auch von europapolitischer Bedeutung.

7 Informationen zu den Living-Wage-Kampagnen in Großbritannien finden sich unter: http://www.livingwage.org.uk/.

8 DerText des „Fair Minimum Wage Act of 2013" findet sich unter: http://fcnl.org/issues/poverty/fair_minimum_wage_act_of_2013/.

\section{LITERATUR}

Aaron, H. et al. (2013): Economist statement on the federal minimum wage http://www.epi.org/minimum-wage-statement/

CDU/CSU/SPD (2013): Deutschlands Zukunft gestalten. Koalitionsvertrag zwischen CDU, CSU und SPD für die 18. Legislaturperiode, http://www.spd. de/linkableblob/112790/data/20131127_koalitionsvertrag.pdf

Fernández-Macías, E./Vacas-Soriano, C. (2013): A coordinated EU minimum wage policy? Eurofound, Draft Version, http://www.eurofound.europa.eu/ docs/press/minimumwages/draftreport_minimumwage_21oct13.pdf Financial Times Editorial (2014): Fairness and the minimum wage, in: FinancialTimes vom 7. Januar

Greater London Authority (2013): A Fairer London:The 2013 Living wage in London, November, http://www.london.gov.uk/sites/default/files/living-wage-2013.pdf

Herr, H./Kazandziska, M./Mahnkopf-Praprotnik, S. (2009): The theoretical debate about minimum wages, Global Labour University Working Papers (6), February

International Labour Organization (ILO) (2013): Global Wage Report 2012/13. Wages and equitable growth, Geneva

Marx, I./Marchal, S./Nolan, B.(2012): Mind the Gap: Net incomes of minimum wage Workers in the EU and the US, IZA Discussion Paper (6510) Plunkett, J./Hurrell, A. (2013): Fifteen years later. A discussion paper on the future of the UK National Minimum Wage and Low Pay Commission, Study of the Resolution Foundation, http://www.resolutionfoundation.org/media/ media/downloads/FINAL_Future_of_the_minimum_wage_discussion_paper. pdf

Schulten, Th. (2012): European minimum wage policy: A concept for wageled growth and fair wages in Europe, in: International Journal of Labour Research 4 (1), S. 85-104

Schulten, Th. (2013): WSI-Mindestlohnbericht 2013 - Anhaltend schwache Mindestlohnentwicklung in Europa, in: WSI-Mitteilungen 66 (2), S. 127-132, http://www.boeckler.de/wsimit_2013_02_schulten.pdf

Schulten, Th. (2014): Mindestlohnregime in Europa ... und was Deutschland aus ihnen lernen kann, Studie für die Friedrich-Ebert-Stiftung, Februar Schulten, Th./Müller, T. (2013): Ein neuer europäischer Interventionismus? Die Auswirkungen des neuen Systems der europäischen Economic Governance auf Löhne und Tarifpolitik, in: Wirtschaft und Gesellschaft 39 (3), S. $291-321$

Veverková, S. (2013): Czech Republic: Minimum wage increase comes into effect, EIROnline, http://www.eurofound.europa.eu/eiro/2013/08/articles/ cz1308019i.htm

\section{AUTOR}

THORSTEN SCHULTEN, Dr., Wissenschaftler im Wirtschafts- und Sozialwissenschaftlichen Institut (WSI) in der Hans-Böckler-Stiftung. Arbeitsschwerpunkte: Arbeits- und Tarifpolitik in Europa.

thorsten-schulten@boeckler.de 